This is the final peer-reviewed accepted manuscript of:

G. Paolini, D. Masotti, F. Antoniazzi, T. Salmon Cinotti and A. Costanzo, "Fall Detection and 3-D Indoor Localization by a Custom RFID Reader Embedded in a Smart e-Health Platform," in IEEE Transactions on Microwave Theory and Techniques, vol. 67, no. 12, pp. 5329-5339, Dec. 2019.

The final published version is available online at: http://dx.doi.org/10.1109/TMTT.2019.2939807

Rights / License:

The terms and conditions for the reuse of this version of the manuscript are specified in the publishing policy. For all terms of use and more information see the publisher's website.

This item was downloaded from IRIS Università di Bologna (https://cris.unibo.it/)

When citing, please refer to the published version. 


\title{
Fall Detection and 3-D Indoor Localization by a Custom RFID Reader Embedded in a Smart e-Health Platform
}

\author{
Giacomo Paolini, Student Member, IEEE, Diego Masotti, Senior Member, IEEE, \\ Francesco Antoniazzi, Student Member, IEEE, Tullio Salmon Cinotti, \\ and Alessandra Costanzo, Senior Member, IEEE
}

\begin{abstract}
In this paper, we describe a customized $2.45 \mathrm{GHz}$ RFID (Radio-Frequency IDentification) reader, designed to simultaneously perform 3-D tracking of multiple tagged entities (objects or people), static or dynamic, in harsh electromagnetic indoor environments. This is obtained by a bi-dimensional electronic beam-steering, implementing the monopulse radar concept simultaneously in the elevation and azimuth directions, with tags-reader distance estimation based on Received Signal Strength Indicator (RSSI) measurements. Experimental results show that the system is able to perform a tri-dimensional scanning of a monitored room with decimeter-accuracy over the three reference axes. The RF front-end is designed to be lightweight, thin and compact in such a way that it is portable and embeddable in domestic objects. For this purpose, a multi-layer solution is adopted with a two-dimensional patch antenna array aperture-coupled with the RF front-end. 3-D localization data are computed on board, by means of a seamless connection of the RF front-end with a low-power microcontroller which is able to store tags 3-D localization data over a multi-hour time frame. A useful method to remotely control the whole system is presented, using a Raspberry Pi 3B, directly connecting the reader with a flexible and extensive digital platform for Smart Homes. The presented architecture is experimentally demonstrated to perform a reliable fall detection of tagged people in indoor environments.
\end{abstract}

Index Terms - 3-D indoor localization, Ambient Assisted Living (AAL), e-Health, remote fall detection, RFID, tracking.

Manuscript submitted May 4, 2019; revised August 3, 2019; accepted August 17, 2019. This paper is an expanded version from the IEEE MTT-S International Microwave Symposium (IMS 2019), Boston, MA, USA, June 27, 2019.

This work was partly supported by the European Commission and the Emilia-Romagna Region, within the framework of the Regional Operative Project "HABITAT" (Home Assistance Based on the Internet of Things for the AuTonomy of everybody).

Giacomo Paolini and Diego Masotti are with DEI "Guglielmo Marconi", University of Bologna, Bologna 40136, Italy (e-mail: giacomo.paolini4@unibo.it,diego.masotti@unibo.it).

Francesco Antoniazzi and Tullio Salmon Cinotti are with DISI, University of Bologna, Bologna 40136, Italy (e-mail: francesco.antoniazzi@unibo.it, tullio.salmoncinotti@unibo.it).

Alessandra Costanzo is with DEI "Guglielmo Marconi", University of Bologna, Cesena 47522, Italy (e-mail: alessandra.costanzo@unibo.it).

\section{INTRODUCTION}

$\mathrm{T}$ HE concurrent use of information and communication technologies, such as Wireless Sensor Network (WSN), Real-Time Localization and Sensing (RTLS), Machine Learning (ML) and Artificial Intelligence (AI) is of great interest for the health care systems. One of the main reasons is that societies are now aging, and many individuals living alone may be subject to disease, to falls, and need to be remotely monitored for their safety. For instance, Alzheimer's disease gradually invalidates many important human capabilities such as memory, reasoning, conceiving and learning. Invisible to family members or doctors, these signs can be recognized by monitoring movements and behaviour of the elderly [1], [2] or by studying the onset of space confusion, often associated with fast repetitive movements in round trips [3].

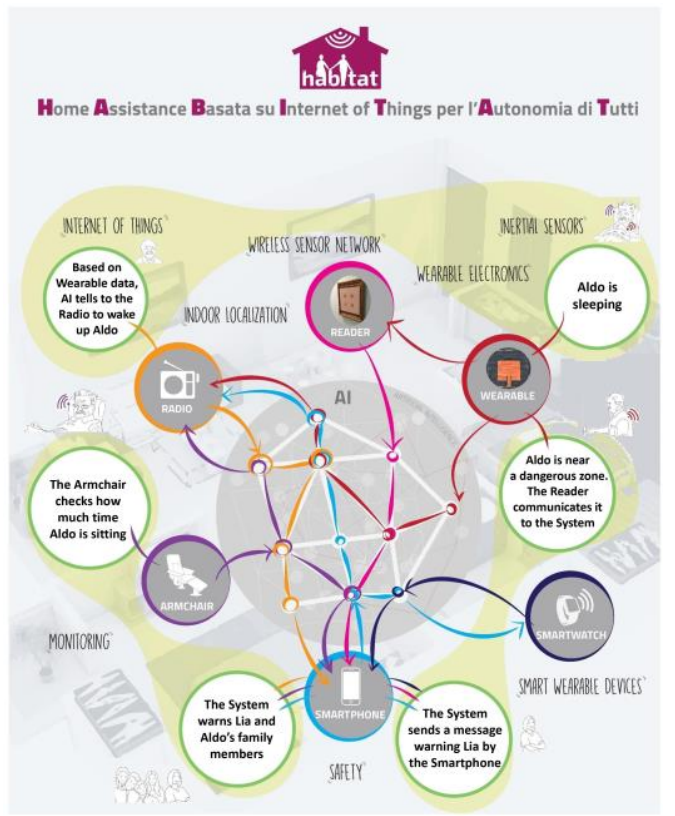

Fig. 1. The "HABITAT" platform: the smart e-Health platform in which the RFID Indoor Localization System is embedded.

Radio-Frequency IDentification (RFID) has demonstrated great potentials with its capability to remotely identify objects 
and people in harsh electromagnetic environments and has been considered in the present work as a suitable solution to the above-mentioned monitoring needs. It is worth mentioning that positioning solutions relying on untagged objects/people are available, too, based on radar technology [4]-[6], but they do not ensure the sensitivity of tagged solutions, as required by the present project for true fall detection.

Several applications of Indoor Positioning System (IPS) based on RFID have been recently proposed to localize patients [7], [8], to detect movements of human body segments [9] and for other smart healthcare applications [10]-[12]. Combination with pan-tilt zoom cameras or accelerometers [13] have also been demonstrated. Ultra-Wide Band (UWB) solutions have demonstrated high accurate localization capabilities being very robust with respect to fading and shadowing that occur in indoor environments [14].

Drawbacks of these available architectures are the need for installing anchor nodes (or readers), that must be synchronized, and not negligible computational time for processing the incoming data.

This work presents an anchorless solution consisting of a portable RFID reader, based on a combined design of the antenna system and the RF front-end, able to simultaneously perform 3-D tracking of multiple tagged entities (static or moving) and to decode the ID of the same active tags. A continuous bi-dimensional electronic beam-steering is enabled on the RFID reader in order to implement the monopulse radar concept and to find the angular positions of the RFID tags, both in the azimuth and in the elevation directions; the tags-reader distance estimation is based on RSSI measurements. In this way an indoor real-time 3-D tracking is available for monitoring not only people movements and occupancy, but also accidental falls.

This system has been embedded in a smart e-Health platform, called "HABITAT" (Home Assistance Based on the Internet of Things for the AuTonomy of everybody) [15], that is illustrated in Fig. 1. It consists of several smart objects developed to monitor elderly people living in their own houses. These objects are interoperable through a unique platform adopting real-time AI procedures to enable decisions and data fusion [16].

The proposed customized RFID system interact in real-time with the e-Health platform to localize tagged people in their daily life environments, prevent their entrance in red zones considered unsafe, control their position and remotely detect their potential falls.

Starting from the 1-D implementation of a reader for objects detection and selection [17], this work expands upon the architecture introduced in [18] and [19], of a $2.45 \mathrm{GHz}$ system with bi-dimensional beam-steering and identification capabilities (Radio-Frequency IDentification). The detailed circuit-level implementation of the 2-D antenna array integrated with a four-way radio circuitry is presented and a multi-layer implementation of the whole system is demonstrated, to provide an agile and fully portable solution. The realized prototype, combining commercial RF chips and customized microwave components, is presented.
Then, the standalone prototype performance is experimentally validated by means of a vast experimental campaign in several realistic indoor scenarios, to establish its operation accuracy in tracking horizontal and vertical tags positions. Measurements show that the system is able to manage simultaneously multiple tagged entities (objects or people), taking advantage of a custom on-board data processing procedure for retrieving their 3-D dynamic position.

Furthermore, a wearable tag is introduced in this work and used for the system validation, to demonstrate seamless tracking of people activities. These measurements show that the presented system can be exploited as a microwave fall detector: it is proved that any possible fall positions are correctly retrieved with no false positive and false negative results, that usually affect other available fall sensors. Important enhancements, with respect to [18], regarding the 3-D localization accuracy, are obtained due to an updated signal processing algorithm coupled with a suitable and fast system calibration in any room before starting the tracking activities.

Finally, details on the on-board implementation of the needed data processing are briefly described together with the integration with the e-Health platform.

\section{RFID READER DESIGN AND REALIZATION}

A customized design of a $2.45 \mathrm{GHz}$ RFID reader (Figs. 2 and 3) has been developed to implement bi-dimensional electronic beam-steering [20] and to take advantage of the monopulse radar concept [21]. The aim is to enable tridimensional localization of tagged people in electromagnetically harsh indoor environments, in the most transparent and non-invasive way. For this purpose, a multilayer architecture of the RF front-end has been chosen with the radiative elements serving as transmitting and receiving antennas, placed at the opposite side of the RF circuitry.

The overall RFID reader board measures $17 \times 17 \mathrm{~cm}^{2}$, and its multi-layered stack-up is shown in Figs. 2: it consists of two external Taconic RF-60A laminates (Inner Layer \#1 and \#2; thickness: $0.635 \mathrm{~mm}, \varepsilon_{\mathrm{r}}=6.15$ ); for mechanical stability reasons, the two Taconic layers are held together through a FR4 film (Prepreg; thickness: $0.120 \mathrm{~mm}, \varepsilon_{\mathrm{r}}=4.5$ ). The top and bottom copper layers metallization (external foils L1 and L4; thickness: $0.035 \mathrm{~mm}$ ) host the antenna array and the RF front-end, including the antenna feeding network and the baseband subsystem, respectively.

For this prototype, the selected working frequency is 2.45 $\mathrm{GHz}$, belonging to the ISM (Industrial, Scientific and Medical) Band (2.4-2.4835 GHz), in order to allow the compactness of the overall system, mainly due to the antenna array, and the centimeter-level accuracy of the localization.

\section{A. Antenna Array Design}

To implement beam scanning in the azimuth $(x y)$ and the elevation ( $y z$ ) planes, a two-dimensional array of patch antennas has been designed and the photo of the corresponding prototype is shown in Fig. 3(a): four squared patches are used, fed by means of rectangular apertures 
obtained in the patches ground planes (L2 and L3 in Fig. 2 (a)), and excited by proper open feeding lines located in the bottom layer (L4 in Fig. 2(a)).

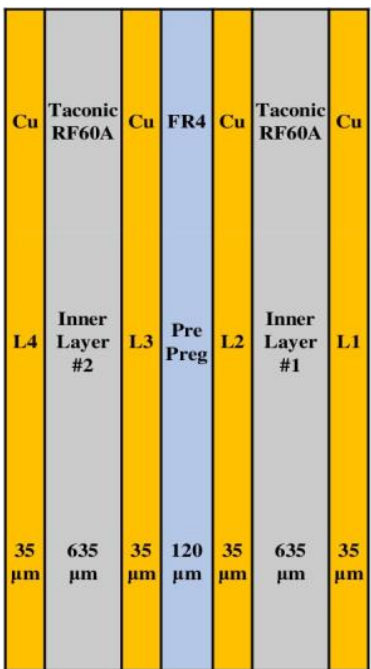

(a)

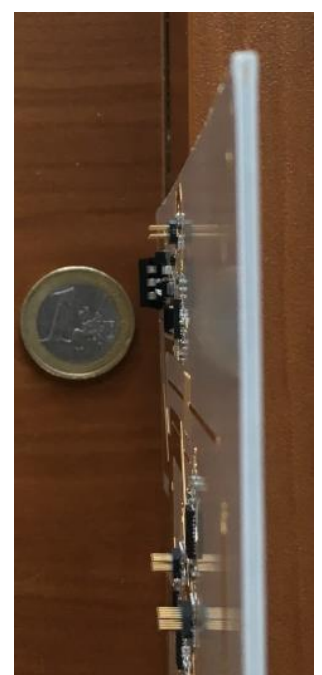

(b)
Fig. 2. (a) Stack-up of the entire board of the RFID reader and (b) picture showing the actual board thickness, measuring $6.15 \mathrm{~mm}$ considering also the ICs. The only RF substrates, prepreg and copper thickness is about $1.53 \mathrm{~mm}$.

The patch array and feeding network optimal dimensions have been obtained after the optimizations accomplished with the electromagnetic (EM) simulation software CST Microwave Studio. With reference to Figs. 3, the optimized quantities have been: i) the length of the square patches $(L=W)$; ii) the patches distances $\left(L_{x}, L_{z}\right)$; iii) the ground aperture dimensions and their relative positions with respect to both the patch in the upper layer (L1) and the feeding line in the bottom layer (L4), see also Fig. 5.

Some representative simulated results of the four-port antenna system (including the feeding lines) are reported in Fig. 4: good matching and low inter-element coupling have been obtained by the present design.

The antenna array feeding network (also visible in the prototype bottom layer of Fig. 3(b)) is schematically depicted in Fig. 5, where the feeding line for each antenna is marked with the corresponding symbols of Fig. 3(b). The microstrip lines width of $0.9 \mathrm{~mm}$ guarantees the $50-\Omega$ characteristic impedance in accordance with both the RF output pin of the transceiver (after the matching network) and the RF output of the phase shifter.

The final layout dimensions are summarized in Table I.

TABLE I

MAIN SYSTEM DIMENSIONS

\begin{tabular}{c|c}
\hline \hline Overall Reader Board Length & $170 \mathrm{~mm}$ \\
\hline Overall Reader Board Width & $170 \mathrm{~mm}$ \\
\hline Overall Reader Board Thickness & $6.15 \mathrm{~mm}$ \\
\hline Patch Length: $\boldsymbol{L}$ & $21.6 \mathrm{~mm}$ \\
\hline Patch Width: $\boldsymbol{W}$ & $21.6 \mathrm{~mm}$ \\
\hline Microstrip Width & $0.9 \mathrm{~mm}$ \\
\hline Slot Length & $1.4 \mathrm{~mm}$ \\
\hline Slot Width & $11.80 \mathrm{~mm}$ \\
\hline Slot Distance from Patch Lower Edge & $4.66 \mathrm{~mm}$ \\
\hline Slot Distance from the Feeding Line Open End & $2.19 \mathrm{~mm}$ \\
\hline
\end{tabular}

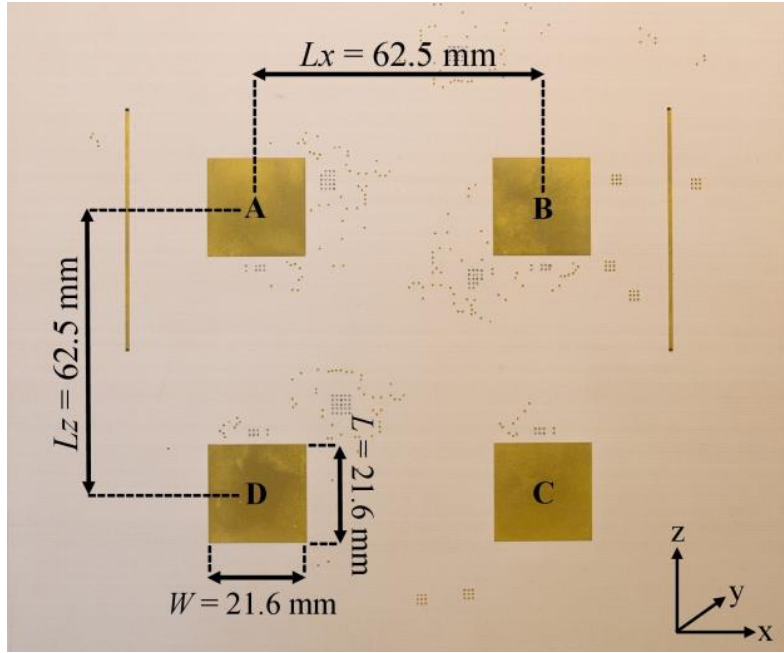

(a)

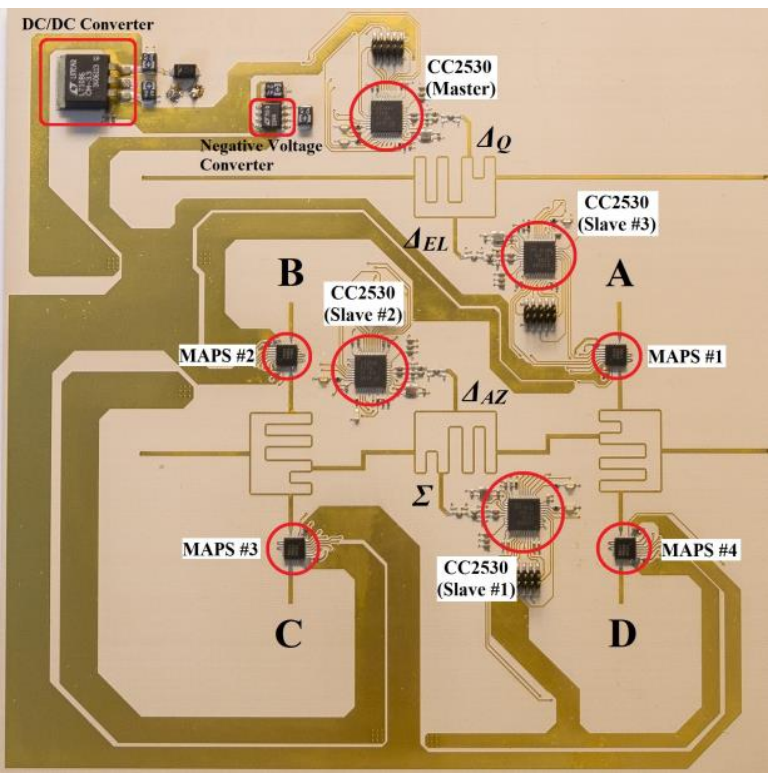

(b)

Fig. 3. Photograph of (a) the top and (b) the bottom layers of the presented RFID reader for 3-D indoor localization. The top layer presents the antenna array, whereas the bottom one contains the digital and RF components and circuits.

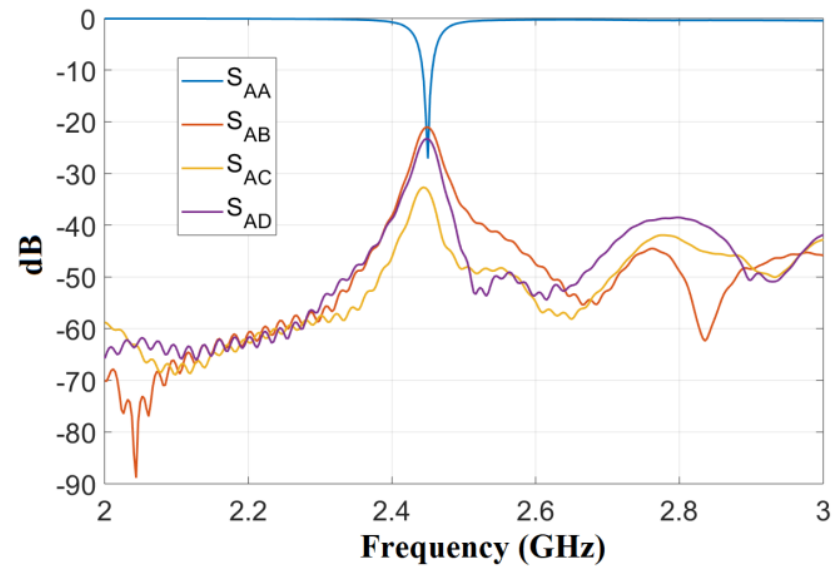

Fig. 4. Scattering parameters of the four-port antenna system (patch antennas and feeding lines). 


\section{B. Master-Slave Operations of the RF Front-Ends}

Subsequently, the 2-D monopulse comparator system, or Rat Race System, has been designed. The schematic layout in microstrip technology is depicted in Fig. 5 and its realization is shown in Fig. 3(b): the open terminations, referred again with the symbols A, B, C, D, are aperture-coupled with the corresponding antennas. The port $\Sigma$ provides the in-phase received signal, $\Delta_{A Z}$ and $\Delta_{E L}$ the out-of-phase ones in the azimuth and in the elevation planes, respectively, and $\Delta_{Q}$ an auxiliary signal [22]. Such signals are processed by the transceivers, marked with the corresponding letters in Fig. 3(b), connected to them.

For this purpose, and to comply with the overall compactness of the system, four meandered rat-race hybrid couplers [17] (represented in Fig. 5) are suitably connected to each other and, at the same time, to the antenna feeding lines and to the RF front-end ports: in this way, the signal combinations superimposed in Fig. 5 are obtained.

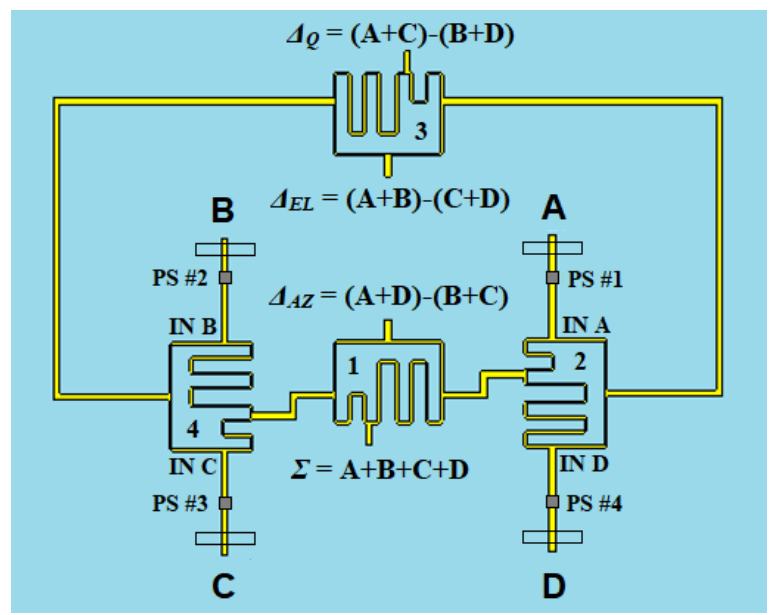

Fig. 5. Schematic representation of the four-port microstrip circuit designed to host the RF and digital subsystems at the system bottom layer (microstrip antenna feeding lines, phase shifters (PS), and monopulse comparator system, or Rat Race System). The $\Sigma, \Delta_{A Z}, \Delta_{E L}$ and $\Delta_{Q}$ ports provide the excitation signals to the transceiver chips. Letters A to D, at the feeding lines ends, correspond with the antenna elements in the upper layer.

The RF hardware architecture and the principle of operation of the whole system are schematically illustrated in Fig. 6.

Four TI (Texas Instruments) CC2530 are used: they adopt a System on a Chip (SoC) configuration, integrating the radio and the microcontroller unit (MCU or $\mu \mathrm{C}$ ) enabling radio communication in the $2.4 \mathrm{GHz}$ band, and are connected to each of the four above-mentioned antenna system ports.

Communication among the four MCUs is established and is based on a Master-Slave logic: the CC2530 Master, connected to the auxiliary port $\Delta_{Q}$, controls, sends and receives information from the three CC2530 Slaves: this is accomplished by the SPI (Serial Peripheral Interface) communication protocol which uses four control signals: i) the Serial Clock (SCLK) to synchronize the whole communication; ii) a number of Slave Select (SS) signals (three for the present case), to activate the proper SoC; iii) Master Input Slave Output (MISO); iv) Master Output Slave Input (MOSI).
In order to perform the dual-axis (elevation and azimuth) tags localization, a bi-dimensional electronic beam-steering of the $\Sigma$ and $\Delta$ radiation patterns is accomplished.

The beam-steering is created by varying the antennas phase excitations through four digital phase shifters (Macom MAPS010164) that are inserted along the antenna microstrip feeding lines and controlled by the corresponding MCU.

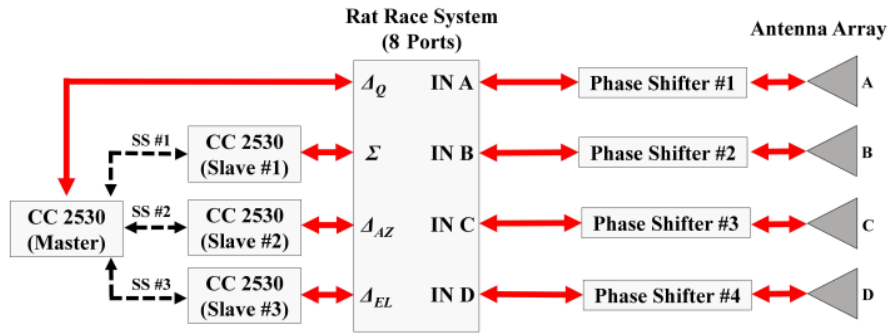

Fig. 6. Block diagram of the hardware architecture and principles of operation (digital I/O and control signals are represented with black dotted lines, while the solid red ones represent the RF signals).

The phase selection is based on a six-bit binary code so that there are 64 possible phase shifts for each phase shifter: to perform a $360^{\circ}$-coverage of the phase, a step size of $5.6^{\circ}$ is used, corresponding to a $1.4^{\circ}$-rotation of the radiation pattern.

Moreover, a DC/DC buck converter (Linear Technology LT1086) and a negative voltage converter (Linear Technology LTC1144) have been introduced in order to guarantee the appropriate supply voltages of $3.3 \mathrm{~V}$ and $-3.3 \mathrm{~V}$, respectively, to the ICs.

The concurrent operation of the master and the slaves SoCs allow to implement in real-time the bi-dimensional beam scanning of the $\Sigma$ and $\Delta$ pattern of the two-dimensional array. To explain the design choices, it is notable to remind that for an array of $n$ identical antennas, aligned along a selected $\hat{a}$ direction, with a constant element spacing $(L)$, the complex current phasor at each antenna element port can be expressed as:

$$
\Lambda_{k} e^{\left(-j \delta_{k}\right)}(A)
$$

where $\Lambda_{k}$ and $\delta_{k}$ are the current amplitude and phase of the array $k$-th element (with $k$ ranging from 0 to $n-1$ ). The array factor, as a function of the elevation and azimuth spherical coordinates, can be cast as follows:

$$
F(\theta, \varphi)=\sum_{k=0}^{n-1} \Lambda_{k} e^{j\left(\beta k L \hat{a} \cdot \hat{r}-\delta_{k}\right)}
$$

where $\beta$ is the phase propagation constant and $\hat{r}$ is the position unit vector. If the phase shift between the currents is constant and equal to $\delta\left(\delta_{k}=k \cdot \delta\right)$, (2) is simplified in the following way:

$$
F(\theta, \varphi)=\sum_{k=0}^{n-1}\left[e^{(-j 2 u)}\right]^{k}=e^{[(n-1) u]} \frac{\sin n u}{\sin u}
$$

where $u=(\pi L / \lambda) \cdot \cos \psi-\delta / 2$ and $\cos \psi=\hat{a} \bullet \hat{r}$. 
The radiation function of the array is then computed from the normalized version of (3), using the radiation function of the array base element $f_{0}(\theta, \varphi)$ :

$$
\begin{aligned}
f(\theta, \varphi)=f_{0}(\theta, \varphi) & \left|\frac{F(\theta, \varphi)}{F\left(\theta_{M}, \varphi_{M}\right)}\right| \\
& =f_{0}(\theta, \varphi)\left|\frac{\sin (n \cdot u)}{n \cdot \sin (u)}\right|
\end{aligned}
$$

where $\left(\theta_{M}, \phi_{M}\right)$ represents the maximum radiation direction of both (3) and $f_{0}$.

For a linear array of two elements, (4) becomes:

$$
f(\theta, \varphi)=f_{0}(\theta, \varphi)|\cos u|
$$

The monopulse radar concept along one direction is realized by an array of two $(n=2)$ antennas in such a way that if $\delta=0^{\circ}$, the two antennas are excited in-phase and their radiation function is of the $\Sigma$-type (i.e., with a maximum in the broadside direction); on the contrary, if they are excited outof-phase $\left(\delta=180^{\circ}\right)$ their radiation function is of the $\Delta$-type (i.e., with a zero in the broadside direction).

To realize two-dimensional $\Sigma$ - and $\Delta$-radiation patterns, four patches aligned along the $x$ and $z$ directions to form a twodimensional array are used, as shown in Fig. 3(a).

The two-dimensional array factor becomes:

$$
f(\theta, \varphi)=f_{0}(\theta, \varphi)\left|\cos u_{x}\right|\left|\cos u_{z}\right|
$$

with:

$$
\begin{gathered}
u_{x}=\frac{\pi L_{x}}{\lambda} \sin \theta \cos \varphi-\frac{\delta_{x}}{2} \\
u_{z}=\frac{\pi L_{z}}{\lambda} \cos \theta-\frac{\delta_{z}}{2}
\end{gathered}
$$

Moreover, with reference to the array feeding network layout of Fig. 5, it can be observed that the excitations for the antenna elements A, B are out-of-phase with respect to those for the elements C, D.

In this way, by a proper control of the current excitations at the antenna ports, namely the phase coefficients $\delta_{i}$ (with $i=\mathrm{A}$, $\mathrm{B}, \mathrm{C}, \mathrm{D})$, the radiation patterns summarized in Table II are made available.

Through the phase shifters, placed in between each antenna feeding line (see Figs. 3(b) and 5), it is possible to electronically vary the relative phases $\left(\delta_{i}\right)$ at each antenna port, in such a way to properly vary the values $\delta_{x}$ and $\delta_{z}$ in (7) between each couple of antennas. In this way, the electronic beam-steering is obtained: depending on the selected phase shifts, it is possible to achieve a scanning in both the $x y$ and $y z$ planes.

TABLE II

Relative Phase ShiFTs of the ANTENNA FeEdings AND CORRESPONDING PATTERNS

\begin{tabular}{c|c|c|c|c}
\hline \hline Pattern & $\boldsymbol{\delta}_{\boldsymbol{A}}$ & $\boldsymbol{\delta}_{\boldsymbol{B}}$ & $\boldsymbol{\delta}_{\boldsymbol{C}}$ & $\boldsymbol{\delta}_{\boldsymbol{D}}$ \\
\hline $\boldsymbol{\Sigma}$ & $0^{\circ}$ & $0^{\circ}$ & $180^{\circ}$ & $180^{\circ}$ \\
\hline $\boldsymbol{\Delta}_{E \boldsymbol{L}}$ & $0^{\circ}$ & $0^{\circ}$ & $0^{\circ}$ & $0^{\circ}$ \\
\hline $\boldsymbol{\Delta}_{\boldsymbol{A} \boldsymbol{Z}}$ & $0^{\circ}$ & $180^{\circ}$ & $0^{\circ}$ & $180^{\circ}$ \\
\hline $\boldsymbol{\Delta}_{\boldsymbol{Q}}$ & $0^{\circ}$ & $180^{\circ}$ & $180^{\circ}$ & $0^{\circ}$ \\
\hline
\end{tabular}

The electronic beam-steering has been simulated in both planes by means of the post-processing tool of CST Microwave Studio and then measured. Representative results of the $\Sigma$ and $\Delta_{E L}$ patterns in the broadside direction and for a $0^{\circ}$ and $15^{\circ}$ down-rotation are compared with the measured ones in Figs. 7. Similar agreement has been obtained for the azimuth plane patterns and the corresponding plots are not reported for the sake of brevity.

Approximately, a $\left[-45^{\circ}, 45^{\circ}\right]$ scan with respect to broadside directions in both the elevation and the azimuth planes has been demonstrated both by measurements and numerical predictions.

In this way, the system is able not only to detect a tag in the horizontal plane, but also to determine its elevation, due to the accurate scanning capabilities along the vertical plane. In this way, the system can be exploited as a portable "people or objects scanner" in 2-D. In particular, it is foreseen to be an interesting device to accurately detect falls of people wearing an active tag.

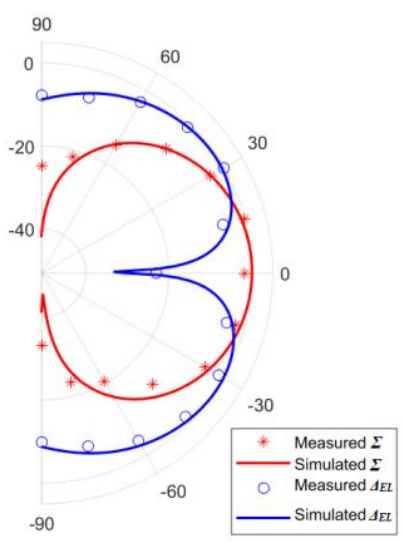

(a)

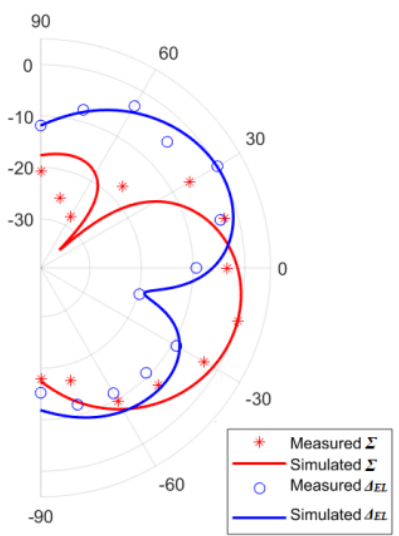

(b)
Fig. 7. (a) Normalized monopulse $\Sigma$ and $\Delta$ radiation patterns (in $\mathrm{dB}$ ) in the elevation plane in the broadside direction; (b) normalized $\Sigma$ and $\Delta$ monopulsesteered radiation patterns (in $\mathrm{dB}$ ) in the elevation plane considering a $-15^{\circ}$ rotation.

\section{MEASUREMENT CAMPAIGNS}

\section{A. Tags Angular Detection Through the Monopulse Radar Technique}

First, the accuracy in localizing the tags angular positions, both in the azimuth and elevation planes, has been tested by Received Signal Strength Indicator (RSSI) measurements at $\Sigma$, $\Delta_{E L}$ and $\Delta_{A Z}$ ports, exploiting the system beam-steering capabilities.

These tests have been carried out in a room, whose photograph is shown in Fig. 8, in the presence of two tags located as follows: i) at 1- and 3-meter distance from the reader and at the same reader height from the ground (125 $\mathrm{cm}$ ), and ii) $30 \mathrm{~cm}$ above and below the reader height.

The $\Sigma$ and $\Delta$ patterns are stored on-board to compute the tags angular positions by means of the Maximum Power Ratio $(M P R)$ computation, for the azimuth and elevation scanning, defined as follows: 


$$
\begin{aligned}
& M P R_{A Z}(d B)=\Sigma(d B m)-\Delta_{A Z}(d B m) \\
& M P R_{E L}(d B)=\Sigma(d B m)-\Delta_{E L}(d B m)
\end{aligned}
$$

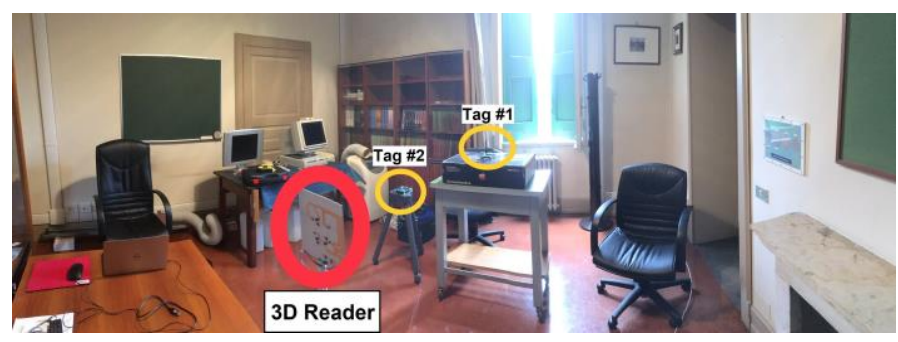

Fig. 8. The indoor scenario where $\Sigma, \triangle, M P R$ retrievals and the first tridimensional localization measurements took place (Villa Griffone, Pontecchio Marconi, Bologna, Italy).

The $M P R_{A Z}$ and $M P R_{E L}$ are used as the system figures of merit in such a way that detecting the tags angular position corresponds to the maximum MPRs.

Measurement results are shown in Figs. 9 and 10 and they clearly demonstrate the system capability to measure the tags elevation and the corresponding azimuth detection, respectively.
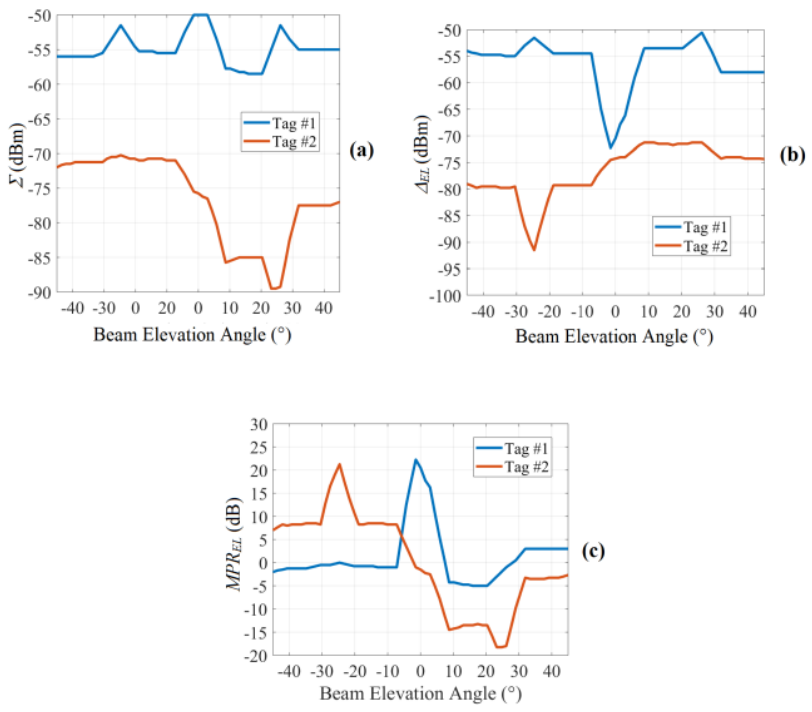

Fig. 9. Elevation detection of two tags: measurements of the RSSI at the (a) $\Sigma$ port, and at the (b) $\Delta_{E L}$ port; (c) resulting $M P R$. The tags are placed at 1-meter (Tag \#1) and 3-meter (Tag \#2) distance from the plane embedding the reader at elevations of $0^{\circ}$ and $-25^{\circ}$, respectively.

\section{B. 3-D Indoor Localization Measurements}

In order to provide a full 3-D localization of tagged people, the collected data from the azimuth and elevation beamsteering operations are further used to predict the tags-reader distance, following a procedure similar to the one introduced in [21]. Once the portable reader is installed in a room, a calibration procedure is first carried out as follows: a virtual reference plane is considered at 1-meter distance from the reader and it is sectorized along the horizontal and vertical directions in such a way that it is divided into zones. The plane dimensions are chosen based on the reader scanning capabilities. For the present case, a plane of $200-\mathrm{cm}$ width (corresponding to a left and right scanning of $67.5^{\circ}$ ) and $60-\mathrm{cm}$ height (corresponding to an upper and lower scanning of $33.4^{\circ}$ ) has been considered, divided in a total of nine zones. Tags are subsequently located in the center of each reference zone and the associated received powers $P_{0 i j}$ are registered, where $i=1, \ldots, N_{A Z}, j=1, \ldots, N_{E L}$, and $N_{A Z}=N_{E L}=3$ are the numbers of horizontal and vertical segments chosen.

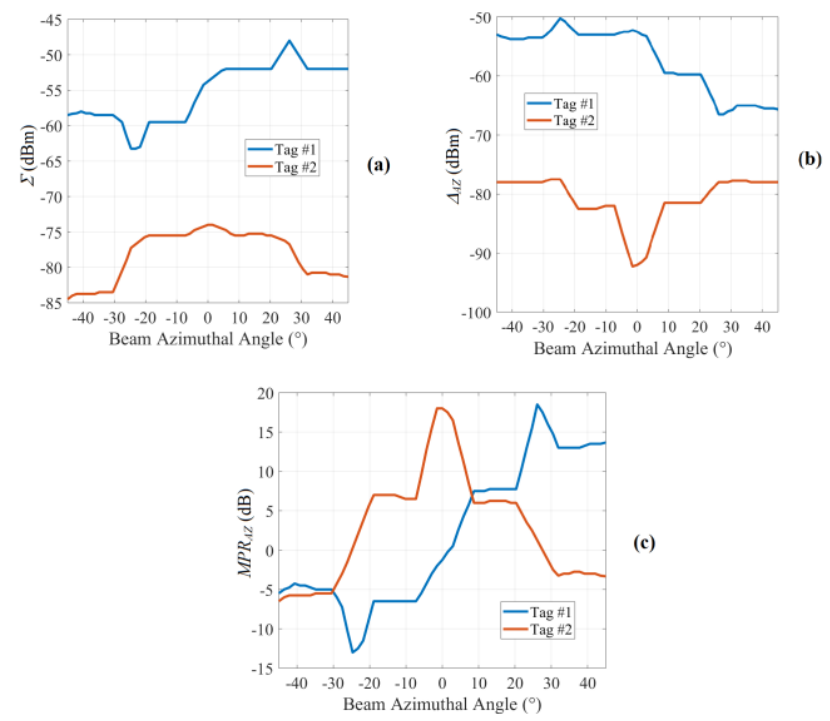

Fig. 10. Azimuth detection of two tags: measurements of the RSSI at the (a) $\Sigma$ port, and at the (b) $\Delta_{A Z}$ port; (c) resulting $M P R$. The tags are placed at 1-meter (Tag \#1) and 3-meter (Tag \#2) distance from the plane embedding the reader at azimuths of $+25^{\circ}$ and $0^{\circ}$, respectively.

Thus, in addition to the azimuth and elevation angular positions, the reader-tag distance $d$ is calculated as follows:

$$
d=d_{0} \cdot 10^{\frac{\left(P_{0 i, j}-P_{R m}\right)}{10 \cdot n_{i, j}}}
$$

where $d_{0}$ is the distance of the reference plane used during calibration, $P_{R m}$ is the actual instantaneous maximum RSSI (in $\mathrm{dBm})$ received at the $\Sigma$-Port, and $n_{i, j}$ are the path-loss exponents for the different calibration zones, depending on the radio channel of the environment under consideration (typical values are: 2.7 to 4.3 for urban areas, 2 for free space, 1.6 for indoor environments) [23], [24]. For the evaluation of these path-loss exponents in a particular scenario, the abovementioned calibration measurements of the RSSI values are replicated at 3-meter distance from the reader, thus deriving the proper $n_{i, j}$ for each calibration zone.

The TI CC2530 radio, integrating the microcontroller, has been used for both the tag side and the four radios of the reader system. For the first set of validation, the CC2530EM evaluation board was used, including the provided monopole as the antenna. The protocol adopted by these radios for the communication is SimpliciTI, the low-power RF network protocol developed by Texas Instruments; this allows to exchange information between the reader and the tags regarding their IDs and the corresponding instantaneous RSSI power values. For these measurements, the same path-loss 
exponent $n_{i, j}=1.9$ for all the calibration zones resulted to be a suitable choice. Some explanatory actual and measured tags positions in the $x y$ plane and the corresponding heights (z-coordinate) are shown in Fig. 11, together with the relative reader location.

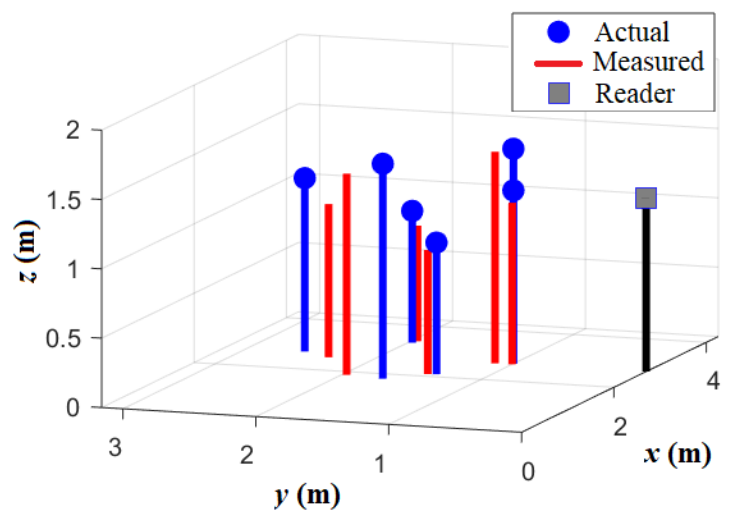

Fig. 11. 3-D localization of six different positions: comparisons between the present system operation and the real positions. For these measurements, the evaluation board CC2530EM was used at the tag side.

A typical laboratory environment (schematically represented in Figs. 13) has been considered as a second scenario. With the aim to validate the whole RFID system for people positioning and monitoring, a wearable antenna has been designed in a denim flexible substrate (dimensions: 5 x 5 $\mathrm{cm}^{2}$, thickness: $1.56 \mathrm{~mm}$ ) and connected to the CC2530 to be worn during the experiments [25], with the ultimate goal of integrating the tag into garments. A simple block diagram of the designed tag architecture is shown in Fig. 12.

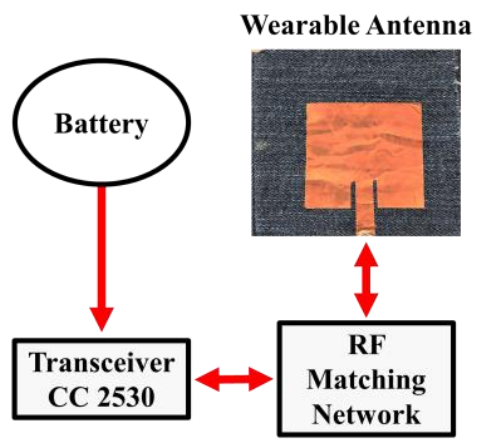

Fig. 12. Block diagram of the adopted RFID active tag (antenna realized on a denim substrate).

Again, for the present case, $N_{E L}=N_{A Z}=3$, so that nine different sectors are used (9-zones calibration). The 3-D indoor localization measurements obtained for ten different tag positions randomly distributed are reported in Table III, together with the errors with respect to the actual locations, that are calculated as follows:

$$
3 \text { Error }=100 \cdot \frac{\sqrt{\left(x_{0}-x_{m}\right)^{2}+\left(y_{0}-y_{m}\right)^{2}+\left(z_{0}-z_{m}\right)^{2}}}{\sqrt{x_{0}^{2}+y_{0}^{2}+z_{0}^{2}}}
$$

Every measurement has been obtained by averaging ten received RSSIs of the same tested point. In this case, the reader is located at a 1.25 -meter height from the ground, a suitable choice for detecting people standing, sitting or falling.

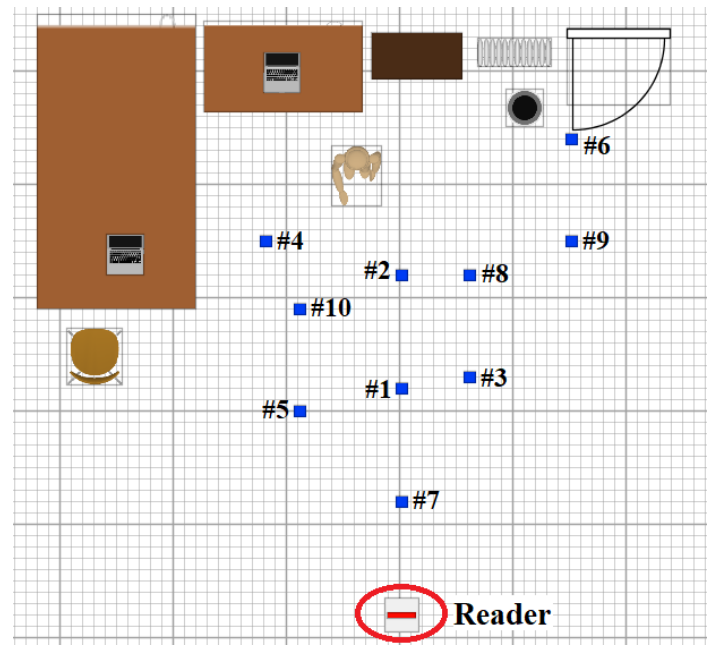

(a)

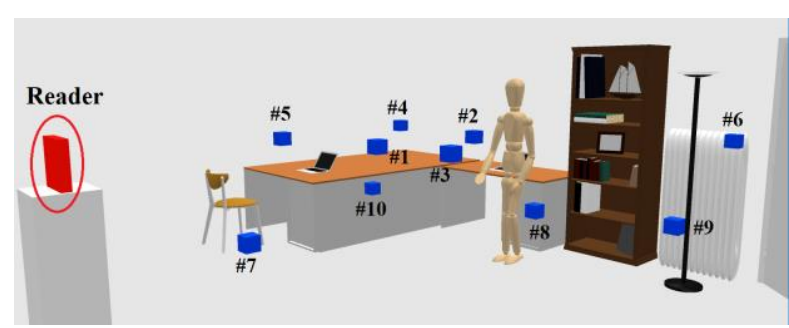

(b)

Fig. 13. (a) 2-D and (b) 3-D location of ten positions considered for the second set of localization measurements (EM Fields Lab, University of Bologna, Italy), with the RFID tags worn by the monitored users.

TABLE III

RESULTS OF 3-D INDOOR LOCALIZATION MEASUREMENTS (WEARABle TAgs, Denim ThickNeSS $=1.56 \mathrm{MM}$ )

\begin{tabular}{|c|c|c|c|c|c|}
\hline \multirow[b]{2}{*}{ Point } & \multirow[b]{2}{*}{$\begin{array}{c}\text { Actual } \\
\text { Position } \\
\left(x_{0} ; y_{o}\right)\end{array}$} & \multirow[b]{2}{*}{$\begin{array}{c}\text { Measured } \\
\text { Position } \\
\left(x_{m} ; y_{m}\right)\end{array}$} & \multicolumn{2}{|c|}{ Tag Height } & \multirow[b]{2}{*}{$\begin{array}{c}\text { 3-D } \\
\text { Error } \\
(\%)\end{array}$} \\
\hline & & & $\begin{array}{c}\text { Actual } \\
\left(z_{0}\right)\end{array}$ & $\begin{array}{c}\text { Meas. } \\
\left(z_{m}\right)\end{array}$ & \\
\hline$\# 1$ & $(5.45 ; 2.00)$ & $(5.47 ; 1.86)$ & 1.25 & 1.25 & 6.00 \\
\hline$\# 2$ & $(5.45 ; 3.00)$ & $(5.30 ; 3.00)$ & 1.25 & 1.25 & 4.62 \\
\hline$\# 3$ & $(6.05 ; 2.10)$ & $(6.07 ; 1.95)$ & 1.25 & 1.17 & 6.80 \\
\hline$\# 4$ & $(4.25 ; 3.30)$ & $(4.46 ; 2.86)$ & 1.25 & 1.38 & 13.54 \\
\hline$\# 5$ & $(4.55 ; 1.80)$ & $(4.70 ; 1.53)$ & 1.25 & 1.20 & 13.21 \\
\hline$\# 6$ & $(6.95 ; 4.20)$ & $(6.37 ; 3.99)$ & 1.25 & 1.02 & 14.21 \\
\hline$\# 7$ & $(5.45 ; 1.00)$ & $(5.40 ; 0.92)$ & 0.75 & 0.73 & 7.71 \\
\hline$\# 8$ & $(6.05 ; 3.00)$ & $(6.34 ; 2.82)$ & 0.75 & 0.77 & 10.85 \\
\hline$\# 9$ & $(6.95 ; 3.30)$ & $(6.45 ; 3.63)$ & 0.75 & 0.97 & 17.24 \\
\hline$\# 10$ & $(4.55 ; 2.70)$ & $(4.92 ; 2.40)$ & 0.75 & 0.67 & 16.41 \\
\hline
\end{tabular}

All Cartesian coordinates and altitudes are expressed in meters. Reader height: $1.25 \mathrm{~m}$. The reader distance from the bottom left corner of the room is $5.45 \mathrm{~m}$. Path-loss exponent $n_{i, j}=1.4$.

To assess the impact of the tag antenna, the same localization scenario of Figs. 13 has been tested using the commercial antenna and two versions of the wearable antenna 
with different thickness of the textile and the results are summarized in Table IV: while with the commercial monopole tags an average error of $20.13 \mathrm{~cm}$ is observed for reader-tag distances lower than $2.60 \mathrm{~m}$, the average error with wearable tags is less than $18 \mathrm{~cm}$ for distances up to $4.50 \mathrm{~m}$.

TABLE IV

AVERAGE LOCALIZATION ERRORS FOR THE THREE MEASUREMENT CAMPAIGNS

\begin{tabular}{c|c|c|c}
\hline \hline Adopted Tag & $\begin{array}{c}\text { Average } \\
\text { 2-D Error } \\
(\boldsymbol{x} ; \boldsymbol{y})\end{array}$ & $\begin{array}{c}\text { Average } \\
\text { Height } \\
\text { Error }\end{array}$ & $\begin{array}{c}\text { Average } \\
\text { 3-D } \\
\text { Error }\end{array}$ \\
\hline $\begin{array}{c}\text { Commercial Tags } \\
(\text { CC2530EM Monopole) }\end{array}$ & $22.70 \mathrm{~cm}$ & $15.00 \mathrm{~cm}$ & $20.13 \mathrm{~cm}$ \\
\hline $\begin{array}{c}\text { Wearable Tag \#1 } \\
\text { (Denim Thickness: } 1.56 \mathrm{~mm})\end{array}$ & $22.20 \mathrm{~cm}$ & $8.30 \mathrm{~cm}$ & $17.57 \mathrm{~cm}$ \\
\hline $\begin{array}{c}\text { Wearable Tag \#2 } \\
\text { (Denim Thickness: } 2.34 \mathrm{~mm})\end{array}$ & $20.30 \mathrm{~cm}$ & $8.50 \mathrm{~cm}$ & $16.37 \mathrm{~cm}$ \\
\hline
\end{tabular}

Finally, the system performance has been tested in the presence of two tagged persons moving in a room, and the tracked trajectories are reported in Fig. 14. They have been moving for 30 seconds, starting from the positions labelled with $t_{0}$, and their measured locations have been compared with the effective ones, which are reported in the same figure: even when the two people were close by, the average 3-D positioning error was under control for the required system specifications to track people activities in a room.

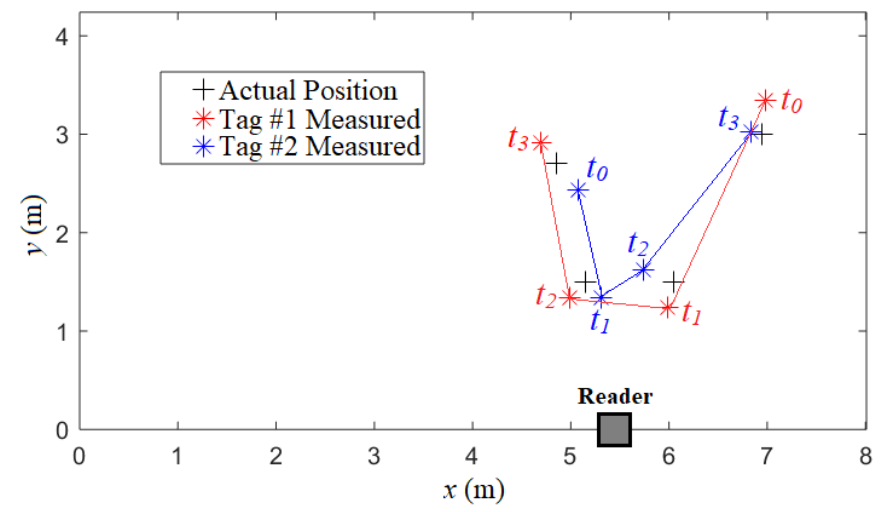

Fig. 14. Actual and measured positions of two tagged subjects moving in the indoor environment during a walk of about 30 seconds. $t_{0}$ is the starting position for each person.

\section{Remote Fall Detection of Tagged People}

The renovated aim of this RFID reader is to detect potential falls that could occur to people living alone in indoor environments.

Exploiting the elevation scanning capability of the RFID reader and its monopulse radar technique, it is possible to notice whenever a tag is fallen or rather positioned on the floor, thanks to the information provided from the $M P R_{E L}$ pattern whose maximum values correspond to the detected elevations of the tags. Moreover, when the tagged person has fallen, his/her height is lower than the reader one, resulting in a lower received power than the one expected when a person is standing: this is detected by the reader and an alert for remote falls can be activated; Figs. 15 and 16 represent the aforementioned conditions, showing $\Sigma, \Delta_{E L}$, and $M P R_{E L}$ for two falling detections of a user wearing the tag, at 1-meter and 3-meter distance from the reader, respectively; it is possible to notice that for the first fall, the tag is out of the calibrated elevation zone of the reader of $\left[-45^{\circ}, 45^{\circ}\right]$, but the system is still able to detect the low tag height, although the $M P R_{E L}$ shows a plateau in the $\left[-45^{\circ},-30^{\circ}\right]$ zone instead of a sharp peak; conversely, for the tagged user lying down at 3-meter distance, the $M P R_{E L}$ maximum peaks is located at $-30^{\circ}$, corresponding to the actual angular position of the active tag in the elevation plane.
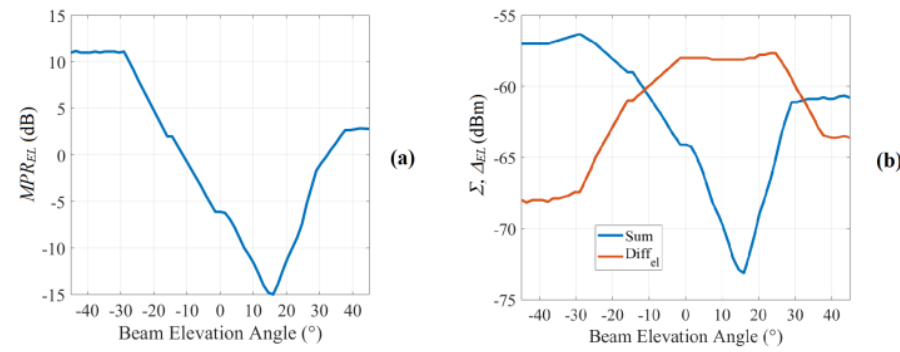

Fig. 15. Diagrams of (a) $M P R_{E L}$, (b) $\Sigma$ and $\Delta_{E L}$ in the elevation plane achieved after the fall of a tagged user at 1 meter of distance from the reader.
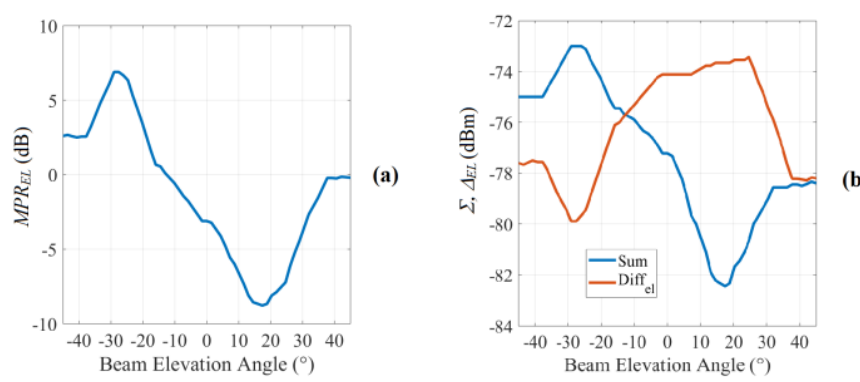

Fig. 16. Diagrams of (a) $M P R_{E L}$, (b) $\Sigma$ and $\Delta_{E L}$ in the elevation plane achieved after the fall of a tagged user at 3 meters of distance from the reader.

Similar results have been obtained for other fall detection experiments in the same indoor scenario of Figs. 13: twelve different fall episodes have been tested and verified: i) lying down face upwards (Fig. 17(a)), ii) face downwards, iii) lateral (on both sides) and iv) sitting (Fig. 17(b)) with readertag distances of $0.5,2$ and 3 meters; these measurements always led to a correct detection of the fall events, with no false positive or false negative situations, demonstrating that the present microwave system can be a reliable solution to detect falls of elderly people living alone, in communities, or cohousing, thus getting rid of continuous assistance.

A further advantage of this proposed fall detection sensor is that it operates alone and in real-time and the reader operations take less than 15 seconds to be completed.

The system can be embedded in everyday objects and wirelessly connected to a smart-home platform augmented with a decision-making algorithm to activate the proper alarms to the proper person.

Indeed, the next Section synthetically describes the seamless integration of the proposed tracking and fall detector system into a general-purpose software platform combining heterogeneous smart objects. 


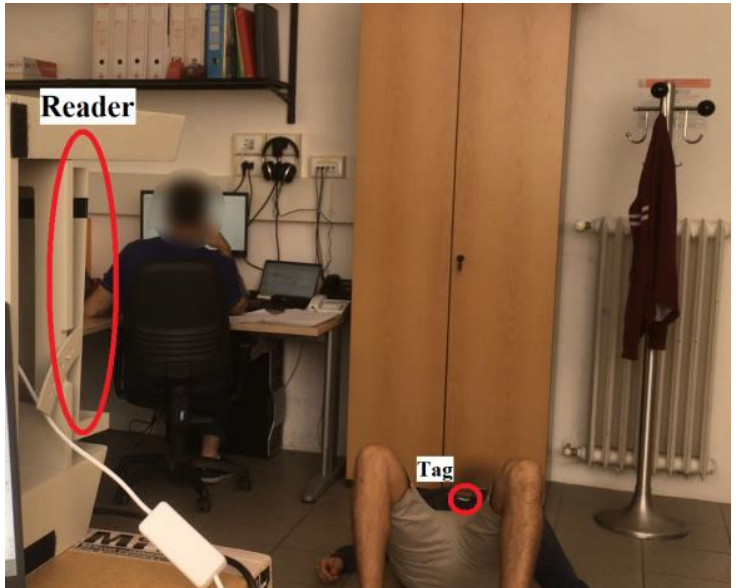

(a)

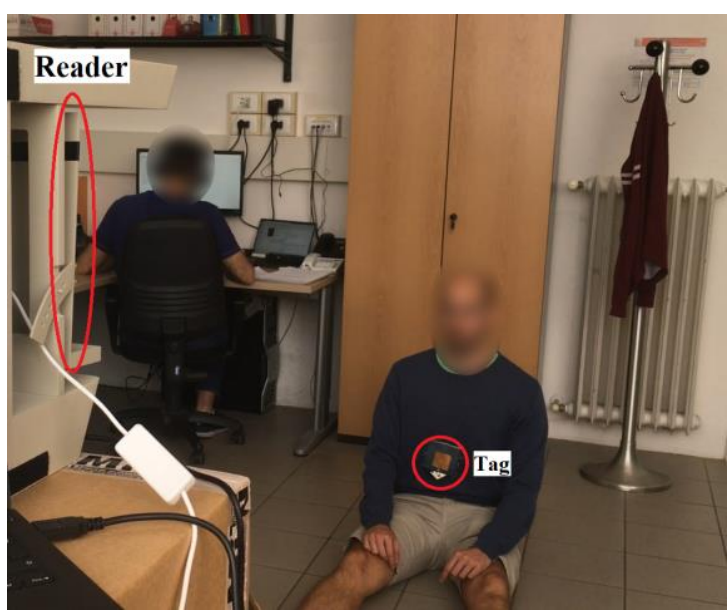

(b)

Fig. 17. Photos of two situations where the reader detects falls: (a) the person is lying down face upwards; (b) the person has fallen down sitting.

\section{DATA REMOTE CONTROL AND ANALYSIS}

Within this Section, the implementation of all the software necessary to use our device into an application is considered.

This is an important task, as the location of people and objects in an environment is a very versatile piece of information useful in a large set of different situations. Smartphones apps and social networks, for instance, perform position reads very frequently, to enhance their services, their advertising, to increase their security policies, etc.

Localization, therefore, is an overall source of Big Data. In our case we deal with the problem of indoor monitoring, but in previous works [26] there is evidence of the possibility to exploit the data coming from the daily routine to achieve ways more than the control of the trajectory of an object or the path chosen by a person.

In such context, the RFID reader is represented as an IoT (Internet of Things) device generating some data that is elaborated by an IoT application [15]. In Fig. 18, a schematic flow chart of the application is outlined: it is composed by a sequence of four tasks whose description is as follows.

First of all (Raw Data step), the system measures the position as it has been explained in Section III. The outcome of the measurement is raw position data that is elaborated in the Analysis and Elaboration phase. A collection of higher- level data, subsequently (Behavioral and Computation phase), is possible by aggregation of this and other information that may be available. Eventually the actual application business logic is possible (Actuation). It is worth noticing that the interaction model for this example is fog-computing [27]: raw data is not immediately sent to external services for elaboration but is rather checked and slightly transformed and cleaned closer to sensors, before the transmission step.

According to this pattern, the reader application for the IoT description follows.

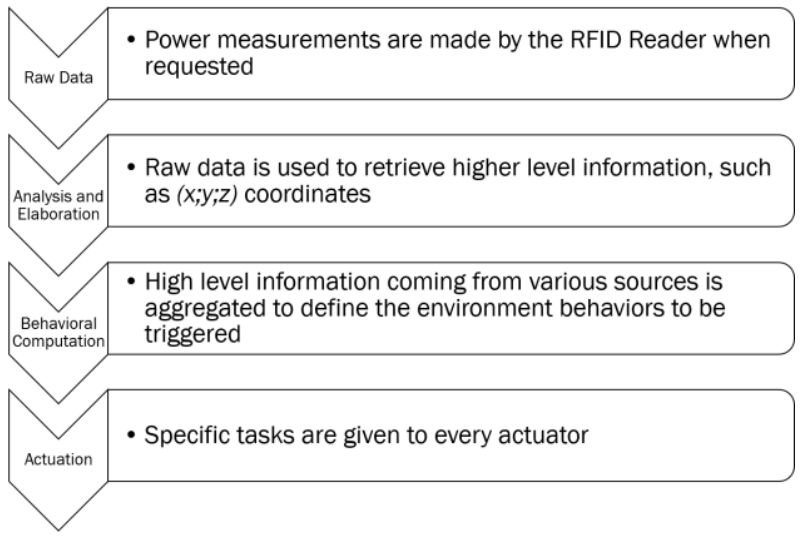

Fig. 18. Abstract scheme for IoT applications in an environment with sensors and actuators.

Phase 1: collect raw data. The firmware is implemented in $\mathrm{C}$ on the TI CC2530 SoC as a simple Mealy-type finite state machine programmed to perform the power scan on the $x y$ plane upon reception of the codified request. Similarly, the protocol also gives the possibility to scan the $y z$ plane, therefore completing the data collection task.

Phase 2: raw data elaboration. Of course, raw data needs to be aggregated into human-readable information and exported to the agents responsible for Phase 3. This task has been performed by a Raspberry PI 3B (RPI3) running a gcc compiled $\mathrm{C}$ program located on board of the RFID reader. It is responsible for the communication with the microcontroller, i.e. they implement all the working protocol to master the scans and dispose of their results.

The communication with the microcontroller has been realized with an UART (Universal Asynchronous ReceiverTransmitter) serial connection. So, eventually, we can sum up the functions of the RPI3 as the following: mastering the microcontroller, reception and elaboration of raw data, export of higher-level data, i.e. the $(x ; y ; z)$ coordinates, to the Phase 3.

Phase 3: behavioral computation, and Phase 4: actuation. The first phase is performed on the IoT server side. When the RPI3 receives all the data and calculates the corresponding $(x ; y ; z)$ coordinates, this information is given to the data sequence aggregation unit located on the server. The communication, in most IoT applications, is made on top of protocols like MQTT, AMQP, CoAP, and others, resulting for every application in a specific and sometimes quite complex stack. Once the new data is received, the server that implements the stack will apply the AI techniques needed for the current application. 
We describe hereby an example application we realized by using a semantic architecture, useful to avoid problems derived from IoT fragmentation [28].

We exploited the semantic publish-subscribe architecture SEPA [29] (acronym of SPARQL Event Processing Architecture) to realize a simple if-this-then-that (IFTTT) application. To do so, the aforementioned RPI3 is then transformed in an information producer, that performs a few triples update in an RDF (Resource Description Framework) store. Those triples, when inserted or removed from the SEPA, trigger a set of information aggregators and consumers. An aggregator might be a (set of) artificial intelligence engine(s), or (some) IFTTT controller(s), while a consumer might be a map visualizing the received location data.

The outcome of this construction, in the end, is the actuation phase. Whenever the AI, or the IFTTT controller detects a need, new triples can be inserted in the SEPA, having the effect of triggering an actuator (or more than one). In our case, as mere example, the IFTTT controller was programmed with a very simple fall-detection algorithm: if the difference $\left|z_{n}-z_{n-1}\right|>T_{z}$, where $T_{z}$ is a threshold, then notify to the caregiver the fall of the person wearing the RFID tag.

\section{CONCLUSION}

In this work, an agile, low-cost and remotely controllable RFID reader operating at $2.45 \mathrm{GHz}$ with real-time 3-D scanning capabilities has been presented and exploited to identify the dynamic position of people moving in an indoor environment. The basics of this system preview the angular scanning of both the azimuth and the elevation planes; moreover, a simple algorithm has been developed to retrieve the reader-tag distance and its height, thanks to the calibration data of the scenario under test and the information coming from the RSSI values, as well as the two angular positions retrieved by the bi-dimensional steering. In this way, an accurate 3-D localization of tagged people in an indoor environment is feasible.

The effectiveness of the 3-D tracking capabilities has been demonstrated by detecting tags positions in indoor scenarios that are typically electromagnetically harsh, in order to early diagnose and care some age-related diseases that are strictly related to the repetition of certain moves.

The accuracy in the tags height detection, with errors lower than $20 \mathrm{~cm}$ in all the experiments adopting the wearable tags, allows to conclude that the present microwave system can be exploited as a novel fall detector in indoor environments, as no false positive or false negative events have ever come across during the system operations.

\section{REFERENCES}

[1] M. F. Mendez, J. S. Shapira, and B. L. Miller, "Stereotypical movements and frontotemporal dementia," Movement Disorders, vol. 20, no. 6, pp. 742-745, Mar. 2005.

[2] H. T. Cheng and W. Zhuang, "Bluetooth-enabled in-home patient monitoring system: Early detection of Alzheimer's disease," IEEE Wireless Commun., vol. 17, no. 1, pp. 74-79, Feb. 2010.

[3] A. Larab, R. Bastide, and B. Rigaud, "Detection of space confusion of people with Alzheimer disease," in Proc. 3rd International Conference on Biomedical Engineering and Informatics, Yantai, China, 2010, pp. 1473-1476.
[4] C. Li et al., "A Review on Recent Progress of Portable Short-Range Noncontact Microwave Radar Systems," IEEE Trans. Microw. Theory Techn., vol. 65, no. 5, pp. 1692-1706, May 2017.

[5] Z. Peng et al., "A Portable FMCW Interferometry Radar With Programmable Low-IF Architecture for Localization, ISAR Imaging, and Vital Sign Tracking," IEEE Trans. Microw. Theory Techn., vol. 65, no. 4, pp. 1334-1344, Apr. 2017.

[6] P. J. Soh, M. Mercuri, G. Pandey, G. A. E. Vandenbosch, and D. M. M. -. Schreurs, "Dual-Band Planar Bowtie Monopole for a Fall-Detection Radar and Telemetry System," IEEE Antennas Wireless Propag. Lett., vol. 11, pp. 1698-1701, 2012.

[7] H. Y. Yu, J. J. Chen, and T. R. Hsiang, "Design and Implementation of a Real-Time Object Location System Based on Passive RFID Tags," IEEE Sensors Journal, vol. 15, no. 9, pp. 5015-5023, Sep. 2015.

[8] H. N. Khan et al., "Measurement based indoor SIMO RFID simulator for tag positioning," in Proc. International EURASIP Workshop on RFID Technology (EURFID), Rosenheim, Germany, 2015, pp. 112-119.

[9] S. Amendola, L. Bianchi, and G. Marrocco, "Movement Detection of Human Body Segments: Passive radio-frequency identification and machine-learning technologies," IEEE Antennas Propag. Mag., vol. 57, no. 3, pp. 23-37, Jun. 2015.

[10] X. Liu, X. Xie, K. Li, B. Xiao, J. Wu, H. Qi, and D. Lu, "Fast Tracking the Population of Key Tags in Large-Scale Anonymous RFID Systems," IEEE/ACM Trans. Networking, vol. 25, no. 1, pp. 278-291, Feb. 2017.

[11] D. Fortin-Simard, J.-S. Bilodeau, K. Bouchard, S. Gaboury, B. Bouchard, and A. Bouzouane, "Exploiting Passive RFID Technology for Activity Recognition in Smart Homes," IEEE Intelligent Systems, vol. 30, no. 4, pp. 7-15, Jul.-Aug. 2015.

[12] L. Catarinucci et al., "An IoT-Aware Architecture for Smart Healthcare Systems," IEEE Internet of Things Journal, vol. 2, no. 6, pp. 515-526, Dec. 2015.

[13] M. B. Akbar, D. G. Taylor, and G. D. Durgin, "Hybrid Inertial Microwave Reflectometry for $\mathrm{mm}$-Scale Tracking in RFID Systems," IEEE Trans. Wireless Commun., vol. 14, no. 12, pp. 68056814, Dec. 2015.

[14] N. Decarli, M. Del Prete, D. Masotti, D. Dardari, and A. Costanzo, "High-Accuracy Localization of Passive Tags With Multisine Excitations," IEEE Trans. Microw. Theory Techn., vol. 66, no. 12, pp. 5894-5908, Dec. 2018.

[15] E. Borelli et al., "HABITAT: An IoT Solution for Independent Elderly," Sensors, vol. 19, no. 5, Mar. 2019.

[16] D. Loreti et al., "Complex reactive event processing for assisted living: The Habitat project case study," Expert Systems with Applications, Elsevier, vol. 126, pp. 200-217, Feb. 2019.

[17] M. Del Prete, D. Masotti, N. Arbizzani, and A. Costanzo, "Remotely Identify and Detect by a Compact Reader With Mono-Pulse Scanning Capabilities," IEEE Trans. Microw. Theory Techn., vol. 61, no. 1, pp. 641-650, Jan. 2013

[18] G. Paolini, D. Masotti, F. Antoniazzi, T. Salmon Cinotti, and A. Costanzo, "Anchorless Indoor Localization and Tracking in Real-Time at $2.45 \mathrm{GHz}$," in Proc. IEEE MTT-S IMS, Boston, MA, USA, 2019, pp. 286-289.

[19] A. Costanzo, D. Masotti, and G. Paolini, "System for locating at least one RFID tag in space, in particular in an indoor environment, and method thereof," Italian Patent 102018000006710, Jun. 27, 2018.

[20] M. Y. W. Chia et al., "Electronic Beam-Steering IC for Multimode and Multiband RFID," IEEE Trans. Microw. Theory Techn., vol. 57, no. 5, pp. 1310-1319, May 2009.

[21] J. C. Wu et al., "Wireless indoor localization using dynamic monopulse receiver," in Proc. 7th European Radar Conference, Paris, France, 2010, pp. $69-72$.

[22] S. Bhilegaonkar and A. Dhande, "Mono-pulse Comparator Network System with hybrid rings," in Proc. 19th Asia-Pacific Conference on Communications (APCC), Denpasar, Indonesia, 2013, pp. 556-560.

[23] Q. Dong and W. Dargie, "Evaluation of the reliability of RSSI for indoor localization," in Proc. International Conference on Wireless Communications in Underground and Confined Areas, Clermont Ferrand, France, 2012, pp. 1-6.

[24] P. C. Liang and P. Krause, "Smartphone-Based Real-Time Indoor Location Tracking With 1-m Precision," IEEE J. Biomedical and Health Informatics, vol. 20, no. 3, pp. 756-762, May 2016.

[25] G. Paolini, D. Masotti, and A. Costanzo, "Wearable RFID Tag on Denim Substrate for Indoor Localization Applications," presented at the 49th EuMC, Paris, France, Sep. 29 - Oct. 4, 2019. 
[26] F. Antoniazzi, G. Paolini, L. Roffia, D. Masotti, A. Costanzo, and T. Salmon Cinotti, "A web of things approach for indoor position monitoring of elderly and impaired people," in Proc. 21st Conference of Open Innovations Association (FRUCT), Helsinki, Finland, 2017, pp. 51-56.

[27] F. Bonomi, R. Milito, J. Zhu, and S. Addepalli, "Fog computing and its role in the internet of things," in Proc. ACM Mobile Cloud Computing Workshop, Helsinki, Finland, 2012, pp. 13-16.

[28] A. Al-Fuqaha, A. Khreishah, M. Guizani, A. Rayes, and M. Mohammadi, "Toward better horizontal integration among IoT services," IEEE Communications Magazine, vol. 53, no. 9, pp. 72-79, Sep. 2015.

[29] L. Roffia, P. Azzoni, C. Aguzzi, F. Viola, F. Antoniazzi, and T. Salmon Cinotti, "Dynamic Linked Data: A SPARQL Event Processing Architecture," Future Internet, vol. 10, no. 4, Apr. 2018.

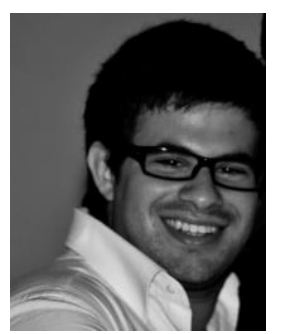

Giacomo Paolini received the M.Sc. Degree in Biomedical Engineering from the University of Bologna, Cesena Campus, Italy, in 2016, and joined the Interdepartmental Center for Industrial ICT Research (CIRI ICT) of the University of Bologna as a Research Fellow within the EU-supported "HABITAT" Project. He is currently with the Department of Electrical, Electronic and Information Engineering " $\mathrm{G}$. Marconi" (DEI) of the University of Bologna where he is working toward the $\mathrm{Ph} . \mathrm{D}$. degree. His research interests include microwave radar systems for biomedical applications, RFID technologies and Indoor Positioning Systems exploiting radiofrequencies.

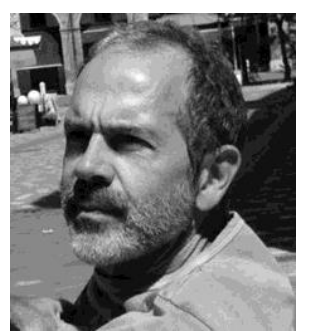

Diego Masotti (M'00-SM'16) received the Ph.D. degree in electric engineering from the University of Bologna, Italy, in 1997. In 1998 he joined the University of Bologna as a Research Associate of electromagnetic fields. His research interests are in the areas of nonlinear microwave circuit simulation and design, with emphasis on nonlinear/electromagnetic codesign of integrated radiating subsystems/systems for wireless power transfer and energy harvesting applications. He authored more than 60 scientific publications on peer reviewed international journals and more than 100 scientific publications on proceedings of international conferences. Dr. Masotti serves in the Editorial Board of the International Journal of Antennas and Propagation, of the Cambridge journal of Wireless Power Transfer, of IEEE Access, and is a member of the Paper Review Board of the main Journals of the microwave sector.

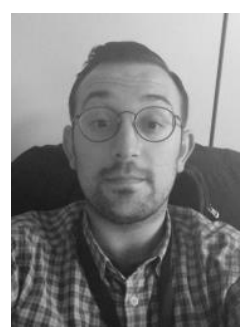

Francesco Antoniazzi was born in Conegliano, Italy, in 1991. He received his B.Sc. in Electronics and Telecommunication Engineering, and then his M.Sc. with honors in Electronics Engineering respectively in 2013 and in 2016 at the University of Bologna, Italy. Currently, he is Ph.D. Student in Computer
Science and Engineering, and Research Fellow at that same University. He has been also visiting Ph.D. student at Queen Mary University of London, and Research Fellow at the Istituto Nazionale di Fisica Nucleare, CNAF section. His research interest is on future Web and IoT technologies.

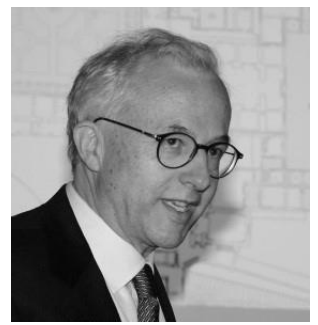

Tullio Salmon Cinotti was born in Bologna, Italy, on May 7th, 1950 and graduated in electrical engineering at the University of Bologna in 1974. He is Associate Professor at the School of Engineering and Architecture of the University of Bologna, and he is in charge of courses on computer architecture and logic design. He was Director of ARCES, an inter-department research center of the University of Bologna in 2015-18. His research interests are in the area of digital systems and go from embedded systems to smart spaces and semantics-based data distribution architectures for cyberphysical systems. He is co-author of researchers from Intel Labs, Nokia Research, Siemens Corporate Technology, STMicroelectronics, Telecom Italia Lab, VTT, Politecnico di Milano, University of Kent. Tullio Salmon Cinotti is coordinator of the University of Bologna participation to European research initiatives in the areas of open cultural heritage, electric mobility, smart agriculture, smart environments and system of systems engineering in the large.

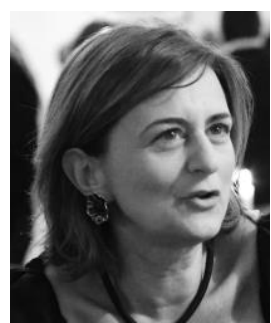

Alessandra Costanzo (A'99-M'02SM'13) is a Full Professor with the University of Bologna, Bologna, Italy. She has authored more than 200 scientific publications in peer-reviewed international journals and conferences and several chapter books. Her current research interests include CAD algorithms for multi-domain co-design and modeling of active nonlinear microwave/RF circuits. Recently, she has proposed novel solutions for energy-autonomous RF systems based on the wireless power transmission, adopting both far-field and near-field solutions, for several power levels and operating frequencies. Dr. Costanzo is the Past-Chair of the MTT-26 Committee on Wireless Energy Transfer and Conversion and a member of the MTT-24 Committee on RFID. She is a cofounder of the EU COST Action IC1301 WiPE Wireless Power Transfer for Sustainable Electronics. Since 2016, she has been the Steering Committee Chair of the IEEE JOURNAL OF RADIO FREQUENCY IDENTIFICATION and is serving as an Associate Editor for the IEEE TRANSACTIONS ON MICROWAVE THEORY AND TECHNIQUES, the Cambridge International Journal of Microwave and Wireless Technologies, and the Cambridge International Journal of Wireless Power Transfer. She is the MTT-S Representative and Distinguished Lecturer of the Council on Radio Frequency Identification (CRFID). She has been TPC co-chair of the MTT-S WPTC2019. 